\title{
X-ray Scattering for the Monitoring of Processes in Polymer Materials with Fiber Symmetry
}

\author{
NORBERT STRIBECK \\ Department of Chemistry, University of Hamburg, Hamburg, Germany
}

\begin{abstract}
Synchrotron facilities with improved equipment will grant the execution of very advanced scattering experiments to every interested polymer scientist. The patterns recorded are two-dimensional, high-resolution, and low-noise images. They permit to monitor structure evolution during the processing of polymer materials with repeat rates of several Hertz. In a different class of novel experiments the structure gradient inside of graded polymer materials will be determined with a spatial resolution of less than $1 \mu \mathrm{m}$. Scattering patterns must be complete. In order both to record complete patterns, and to evaluate them within tolerable time it appears reasonable to study parts with fiber symmetry. In the present paper a review of corresponding methodical work and the results of test experiments is presented that has recently been compiled in the group of the author.
\end{abstract}

Keywords polymers, fibers, WAXD, SAXS, tomography

\section{Introduction}

Presently we are experiencing that intense, reliable X-ray sources and fast detectors become generally available. At DESY in Hamburg PETRA III will provide one of the most brilliant $\mathrm{X}$-ray sources worldwide. There less than a second will be sufficient to expose a lownoise two-dimensional (2D) scattering pattern of a polymer sample. Moreover, in detector development a similar breakthrough has been achieved. Cycle times of less than a second can easily be realized with the novel PILATUS ${ }^{1,2}$ detectors.

It may be objected that for a long time low-noise scattering curves can be recorded with repeat frequencies of several Hertz. Nevertheless, the value of those older data is limited. Either the material is exhibiting isotropic scattering - in this case a comprehensive analysis requires assumptions on the structure (e.g. the assumption of an ideal lamellar stack). Or the material is anisotropic - then a measured curve does not describe the complete scattering pattern or the old 2D pattern is noisy. On the other hand, by application of the new technology it will become possible for many polymer scientists to monitor the structure evolution in oriented polymer materials in real time. The results of such experiments offer the potential to strengthen the understanding of structure evolution mechanisms in polymers. Moreover, the new instruments provide very narrow X-ray beams with diameters of less than $1 \mu \mathrm{m}$ ("microbeams") that permit to study the variation of structure inside the material with respective spatial resolution. A complete sweep of a polymer fiber by a microbeam

Received September 21, 2009; accepted November 5, 2009.

Address correspondence to Norbert Stribeck, Department of Chemistry, University of Hamburg, D-20146 Hamburg, Germany. E-mail: norbert@ stribeck.de 
will take ca. $30 \mathrm{~s}$. Thus, spatial resolution and time resolution may be combined to study the response of different annular zones in a polymer fiber to, e.g. mechanical or thermal load.

Parts with fiber symmetry, like fibers, rods, or tubes, are not only of practical interest because they are ubiquitous in everyday life. Moreover, they appear particularly suited for investigation by means of scattering methods. As the part is rotated about its axis, the $2 \mathrm{D}$ scattering pattern does not change. Thus, there is no need to take patterns at different rotation angles, as it would be necessary for materials with less symmetry. The recorded $2 \mathrm{D}$ scattering pattern of a fiber is oriented and contains all the accessible information on the structure of the sample.

Nevertheless, it will not be sufficient to simply engage the novel instruments-the anticipated data must be adequately evaluated in due time, as well. In this connection two big problems are arising. On the one hand, the data flood is increasing to such an extent that automated evaluation methods must be developed. On the other hand, the data structure has changed, in principal. Instead of scattering curves scattering images must be evaluated. In fact, reasonable scattering curves can be extracted from scattering images as well, ${ }^{3}$ if loss of information is accepted. Nevertheless, it appears more reasonable to develop evaluation methods adapted to the processing of images as a whole. This goal can be achieved by combining digital image processing and scattering theory. ${ }^{4}$ In this review several methods are presented that have recently been developed in our group in order to quantitatively evaluate extensive sets of $2 \mathrm{D}$ scattering patterns from polymer materials with fiber symmetry. Two previously published reviews deal with other aspects of the scattering experiments from polymer materials, namely a presentation of scattering theory ${ }^{5}$ from the point of view of the materials scientists and a presentation of instrumental development. $^{6}$

\section{Practice of Experiment and Data Analysis}

It is not seldom that users who have carried out experiments at a synchrotron source return with incomplete data. In the worst case only images (the TIFF files from the detector) have been collected and relevant environmental data are missing. Such data are the primary beam intensities before and after the sample, exposure time, exposure mode, time stamp, etc. More frequently the user has forgotten to record a machine background or parameters of the setup are incomplete. Such errors could be reduced if the operator of the synchrotron source would offer a data pre-evaluation service. The required data and the steps of data preevaluation are described in a text book ${ }^{4}$ of the author. With respect to the description given in the book the procedure of machine-background elimination has recently been changed so that it becomes consistent with tomographic experiments, as well. Now the intensity in the scattering pattern is divided by the linear absorption factor of the sample and from this image the measured machine background is subtracted. This method compensates intensity loss by absorption inside the sample.

For the professional analysis of anisotropic $2 \mathrm{D}$ scattering patterns of polymer materials there are no user-friendly standard computer programs, because materials scientists do not carry out standard experiments (like, e.g. protein crystallographers). Therefore some program modules must be adapted to the experiment in order to reflect the actual setup or the actual mode of operation. After adaption and proper combination of modules the data can be evaluated automatically. The other option is cumbersome frame-by-frame manual evaluation that is prone to inconsistency or incompleteness. 
The sequence of evaluation steps is a function of several parameters like the setup, the individual scattering power of the studied material, and the features of the anisotropic scattering pattern. New program modules must be constructed if the experimental procedure is fundamentally changed. Complex correction modules may be required if experimental shortcomings are detected during data evaluation. A typical shortcoming is insufficient synchronization among the different recorders of the experiment. Thus, the acquisition of programming skills is recommended. Moreover, it is helpful to choose a computing language that is optimized for the processing of multidimensional data. Suitable commercial platforms are, e.g. PV-WAVE, IDL or MatLab. If free programming tools shall be used, ImageJ offers a good starting point for arduous programmers. Actual references are readily found by search engines on the worldwide web. Our group uses PV-WAVE. ${ }^{7}$ The sources of the modules are free and available. ${ }^{8}$

\section{WAXD Fiber Mapping}

\subsection{Motivation and Method Design}

In wide-angle X-ray diffraction (WAXD) experiments the diffraction patterns must be mapped into reciprocal space before they can be analyzed quantitatively. For this purpose interactive computer programs ${ }^{9,10}$ are utilized that rest upon unnecessary ${ }^{11}$ approximations. Such a design is no disadvantage in crystallography, because sophisticated interactive refinement methods are required anyway for the exact determination of crystal structure parameters in manageable series.

In contrast, in materials science frequently time-resolved experiments are carried out, and voluminous series of diffraction patterns must be processed. The materials scientist already knows the unit cell parameters. Thus, minor inaccuracy of the mapping can be tolerated, if in the experiment variation of peak intensity or shape shall be monitored. Here it is important to carry out the mapping fast and automatically. Because the fiber tilt may change during the experiment, the algorithm must be able to track and to compensate such variation. By revisiting the theoretical treatment of the fiber mapping it has been demonstrated ${ }^{11}$ that there is no principal reason to refine an approximate center of the fiber pattern iteratively. Moreover, instead of an approximation ${ }^{12,13}$ of the tilt angle $\beta$ of the fiber an exact equation ${ }^{11}$ can be employed. In the methodical paper ${ }^{14}$ an algorithm is presented by which the mapping can be performed automatically. Its design rests on the application of the mentioned findings. Intricate parametrization is simplified, and slow trigonometric functions are avoided to a large extent. The method is unsuitable for diffuse scattering patterns. If inaccuracies of 2 pixels can be tolerated, a pattern is automatically mapped into reciprocal space in real time.

\subsection{Actions Required by The User}

For each series of diffraction patterns from a time-resolved experiment, some mapping parameters must once be determined interactively. Our procedure wf_premap assumes that the studied material exhibits a sharp reflection that is located neither on the equator nor on the meridian. In the example we investigate polypropylene and select the (131) reflection of the crystallographic $\alpha_{2}$-modification as the internal standard. With the crystallographic c-axis parallel to the meridian, the reflection is characterized by the parameters ${ }^{15} d_{h k \ell}=$ $d_{131}=0.406 \mathrm{~nm}$ and by $c / \ell=0.6504 \mathrm{~nm}$ that defines the position of the reflection ring 

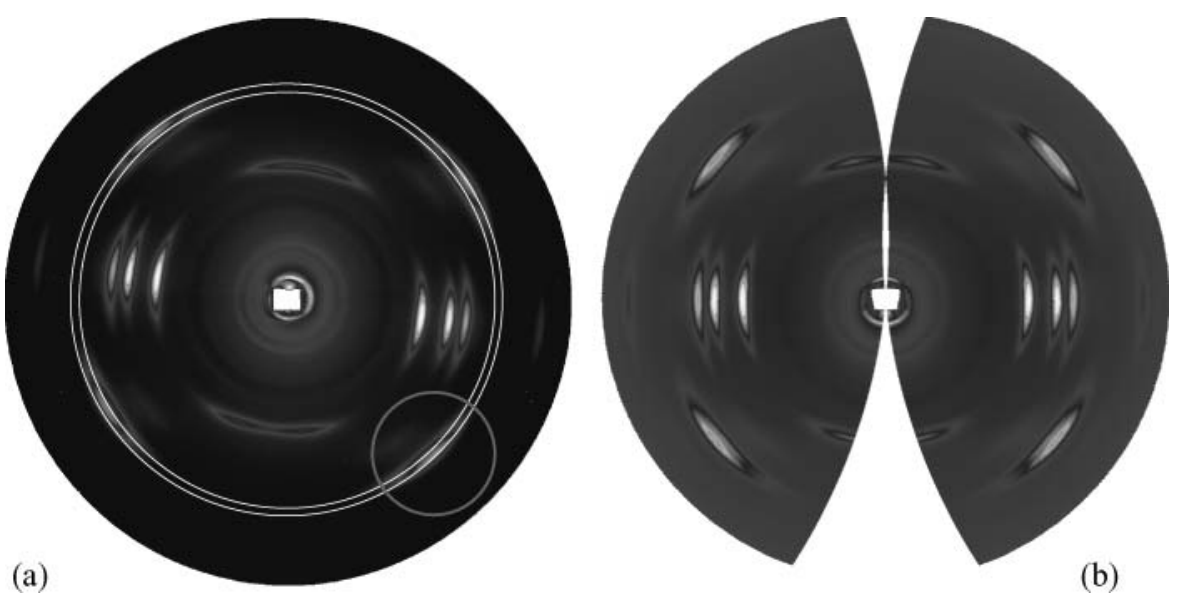

Figure 1. (a) Interactive mode of wf _premap: Draw the reflection circle through the centers of the reflection spots of an internal standard reflection (here: polypropylene (131) reflection), and widen the circle into a belt that contains the maxima of the reflection spots. Finally input 4 circles (one indicated at bottom right). (b) The procedure wf map has mapped the fiber diffraction pattern into reciprocal space.

on the Polanyi sphere. ${ }^{11,16,17}$ The wavelength of X-radiation (here: $\lambda=0.15 \mathrm{~nm}$ ) must be known, too. In this case the first pre-mapping run of the series is started by

wave $>a b=w f \_p r e m a p(s s, a, 0.15,0.406,0.6504)$

The procedure requires a diffraction pattern as input (a). It generates output both in a "saveset" (ss), and in a background-corrected diffraction pattern ( $\mathrm{ab})$. If the provided save-set has never been used before, the procedure enters interactive mode and the user is presented the pattern as shown in Fig. 1.

Obviously, in Fig. 1a the meridian is not vertical and the fiber is tilted. By means of the pointing device the user draws the reflection circle through the maxima of the reflection spots of the (131)-reflection. Thereafter he transforms the circle into a belt that is wide enough to contain the maxima of the spots (Fig. 1a). Now the procedure cuts out this belt and presents it to the user, who finally specifies 4 disjoint clips (regions of interest on the reflection circle). This is done by punching out circular regions from the belt (the bottom right one is shown in Fig. 1a. The clips are the intersections of the belt (white double-band) and the smaller circles. The program determines the positions of the maxima in the clips. From these 4 maxima-positions the best reflection circle is determined by regression. The error of determination is computed. In general, it is below 1 pixel. After that the orientation of the meridian is computed both from the upper and from the lower pair of spots. The difference is, in general, approximately $0.1^{\mathrm{O}}$. Based on this information the diffraction pattern is centered and aligned. Finally, the program computes the tilt angle $\beta$ of the fiber from the orientation angles ${ }^{11} \delta$ and $\delta^{\prime}$ of the spots using the exact equation ${ }^{11}$

$$
\tan \beta=\frac{\sqrt{4-\lambda^{2} s_{r}^{2}}}{2 \lambda s_{r}}\left(\cos \delta^{\prime}-\cos \delta\right) .
$$

Here $s_{r}=1 / d_{131}$ is the radius of the Polanyi sphere of the reference (131)-reflection. Finally, the save-set ss is filled with several data: the tilt angle $\beta$; the true radius $p_{r}$ 
of the reference-reflection circle on the detector; the crystallographic data of the reference reflection; the wavelength $\lambda$; and the geometrical data of the 4 clips. This information is sufficient for an automatic processing of the complete series of diffraction patterns recorded in a time-resolved X-ray diffraction experiment of materials with fiber symmetry.

\subsection{Automated Mapping}

If the procedure wf_premap is called with a filled save-set, the interactive part is skipped, and the geometry of the 4 clips is taken from the save-set. For each clip the position of the spot maximum is computed. From these positions the fiber tilt angle $\beta$ is computed, and the image is centered and aligned. Finally, the mapping into reciprocal space is accomplished by the routine wf map. The function call

wave $>$ rec $=w f \operatorname{map}(s s, a b)$

maps the diffraction pattern ab into reciprocal space using the save-set ss. The result is called rec. A more detailed description is in the original paper. ${ }^{14}$ Figure $1 \mathrm{~b}$ shows a typical result obtained by automatic direct mapping. In the example the computed tilt angle is $\beta=5.85^{\circ}$. Because the result pattern is in reciprocal space, it should exhibit symmetry in 4 quadrants. Thus, the quality of the mapping can be assessed by comparison to a 4-quadrant average of the pattern.

\subsection{Application}

In a study ${ }^{18}$ that applies the method, uniaxially oriented polypropylene (PP) is molten and crystallized isothermally from the oriented, quiescent melt. The results show that nucleation and growth of differently oriented sets of crystallites ( $c$-set and $a^{*}$-set) are decoupled. After shallow quench crystallization is preceded by (spinodal) decomposition. Peak integrals (crystallinity) and minimum crystallite size are tracked. In the commercial starting material $a^{*}$-set crystallites melt at $158^{\circ} \mathrm{C}$. The $c$-set melts at $170^{\circ} \mathrm{C}$ furnace temperature. After recrystallization both sets melt at $170^{\circ} \mathrm{C}$. Isothermal crystallization is divided in two distinct phases. During nucleation the crystallinity stays low. The second phase is dominated by crystallinity growth. At $150^{\circ} \mathrm{C}$ the $c$-set is seeded first. At $145^{\circ} \mathrm{C}$ and $140^{\circ} \mathrm{C} a^{*}$-oriented crystallites are the first. The first-seeded set starts to grow first, as well. $c$-set crystallinity is always growing faster than $a^{*}$-set crystallinity. The evolution of the corresponding SAXS ${ }^{19}$ cross-diagram in the growth phase can both be explained by lamellae growing at right angles, and by block merging.

Figure 2 shows $\beta(t, T)$ of one of the experiments. The tracking curve appears smooth and demonstrates the reliability of the tilt-angle determination. Tilt-angle variation is an issue, because the oriented PP film is heated until it becomes a viscous melt. Therefore the material shrinks and bends in the synchrotron X-ray beam.

After the mapping the intensity distribution is known almost everywhere in reciprocal space except for a wedge region near the meridian (cf. Fig. 1b). Thus, reflection intensities can readily be integrated in reciprocal space. In reciprocal space the relation between scattering and structure is clear from scattering theory, ${ }^{4,18}$ and structural data can be computed, ${ }^{18}$ e.g., from total intensities (crystallinity) and integral breadths (crystallite sizes).

Figure 3 shows the evolution of the weight crystallinities of the two sets of crystallites at 3 different crystallization temperatures. As shown by Ruland, ${ }^{20}$ such reflection integrals that 


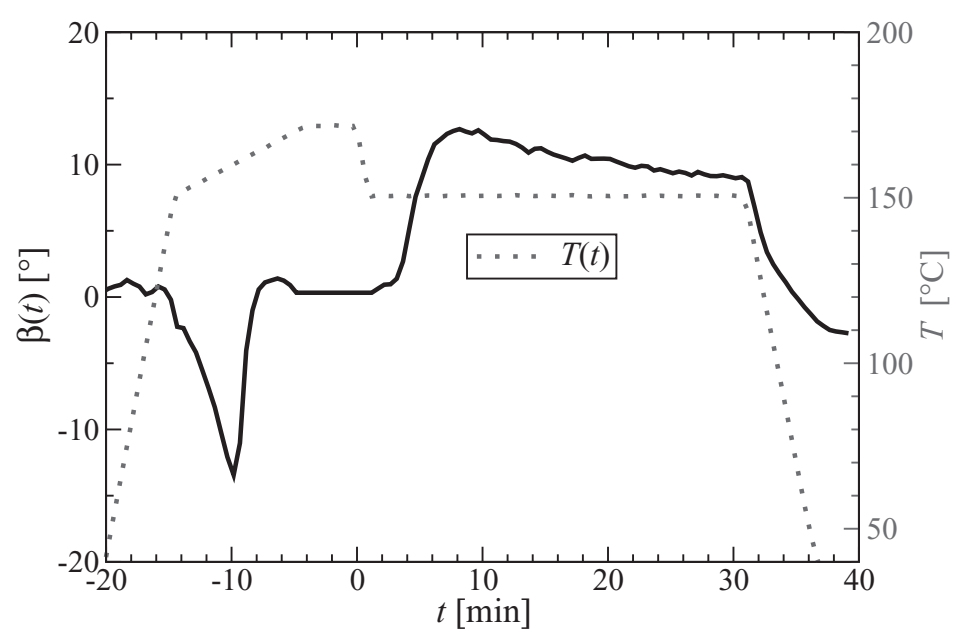

Figure 2. Tilt-angle tracking curve $\beta(t, T)$ from the automatic fiber-mapping procedure in an experiment in which $\beta$ is changing considerably (hard-elastic polypropylene; melt-annealing at $171^{\circ} \mathrm{C}$ and recrystallization at $150^{\circ} \mathrm{C}$ ) (C) Reproduced ${ }^{18}$ with permission of the ACS).

are complete in reciprocal space are proportional to the weight crystallinity of the perfect crystallites that produce the reflections. Latency periods between the quench and the start of the crystallization, as well as crystallization velocities of the two kind of crystallites can be extracted from these data. Finally, conclusions concerning the crystallization mechanisms can be drawn. ${ }^{18}$

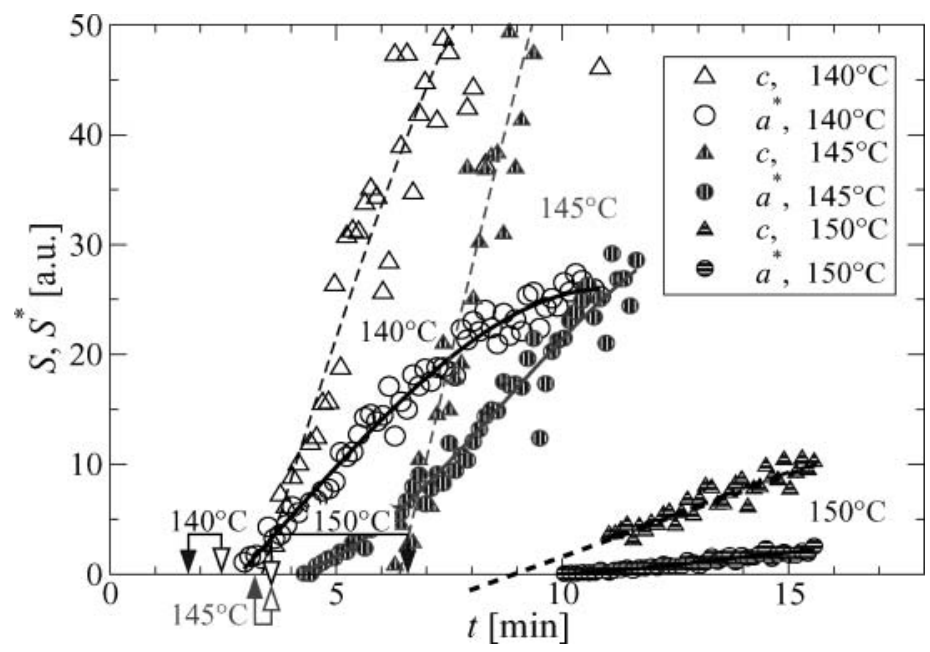

Figure 3. Evolution of relative weight crystallinities $S$, and $S^{*}$ of $c$-oriented crystallites (dashed regression lines) and $a^{*}$-oriented crystals (solid regression lines), resp. during isothermal, oriented crystallization of hard-elastic PP from a quiescent melt as a function of crystallization temperature. Double-head arrows pointing at the $t$-axis indicate the first sighting of the $a^{*}$-set (full arrow head), and of the $c$-set (open arrow head), respectively. 


\section{X-Ray Scattering Fiber Tomography}

\subsection{Motivation}

The structure inside a polymer part is not necessarily homogeneous. With fibers, extrudates, and latex particles ${ }^{21}$ frequently a core-shell structure is reported. Moreover, engineers deliberately generate structure gradients in polymer parts for special functionality. ${ }^{22,23}$ If such a part is irradiated by an X-ray beam, the recorded pattern is an integral superposition of all the SAXS patterns emerging from the sequence of volume elements (voxels) along the beam path. From the mathematical point of view such a superposition is a projection, and a single projection is of little use for the study of graded materials.

A first step towards a study of structure gradients has been the development of the X-ray microbeam technique. ${ }^{24-26}$ Here only the diameter of the beam is limiting the lateral spatial resolution. Nevertheless, the longitudinal spatial resolution is simply the thickness of the sample. Microbeam scanning experiments, in particular of fibers, have been performed for many years and the raw data have been discussed, although the corresponding shortcoming has been known (Paris et al.:27 "The long-term goal is to proceed from microbeam scanning experiments to a real imaging technique"). The solution of the problem is tomographic reconstruction. Problems arise from the fact that scattering patterns are multidimensional but not simply a number (like the absorption in classical tomography). Thus, approximate tomographic reconstruction of scattering data with a manageable amount of artifacts is difficult. An exception is the case of part with uniaxial symmetry. In this case a reconstruction of the scattering patterns is possible that would have emerged from individual voxels in a plane perpendicular to the "fiber" axis. ${ }^{28}$ Nevertheless, this method is only of academic value. The exposure time for the recording of scattering data of one cross-section of the part is in the order of days. The computing time for the tomographic reconstruction of the scattering patterns is at least a week. ${ }^{28}$

\subsection{Introduction of the Method}

A more practical tomographic method can be applied, if the part to be studied both exhibits macroscopic fiber symmetry, and the structure only varies as a function of the distance from its central axis. By means of this method fibers, pipes, and extruded strands can be investigated. Thus, we call it "X-ray scattering fiber computer tomography" (XSF-CT). A complete set of projected scattering patterns is collected in a single microbeam scan across the fiber, because the set of projections does not change as the sample is rotated about its axis. Such an experiment is completed in about $30 \mathrm{~min}$. Moreover, compared to the general tomography the mathematics of image reconstruction is simplified considerably and the computational effort decreases by 5 orders of magnitude. A set of 40 measured scattering patterns is reconstructed in typically less than $10 \mathrm{~min}$. From medicine and other fields of science the considerable potential of information increase after tomographic imaging is well-known.

In a general tomographic X-ray experiment, ${ }^{29}$ a voluminous sample is scanned by a thin $\mathrm{X}$-ray beam. As a function both of the position $x$ of the scanning beam on the sample, and of the sample rotation angle $\phi$, projections (notation: \{\}$)$ of the absorption $\{A\}(x, \phi)$ or even of complete scattering patterns $\{I\}(\mathbf{s}, x, \phi)$ are measured, in order to analyze the structure variation in the plane of the sample that is scanned by the X-ray beam. ${ }^{28,30,31}$ Here $\mathbf{s}$ is the scattering vector with $|\mathbf{s}|=s=(2 / \lambda) \sin \theta$, the X-ray wavelength $\lambda$ and the scattering angle $2 \theta$. In the examples mentioned, a tomographic image reconstruction ${ }^{29,32,33}$ returns 

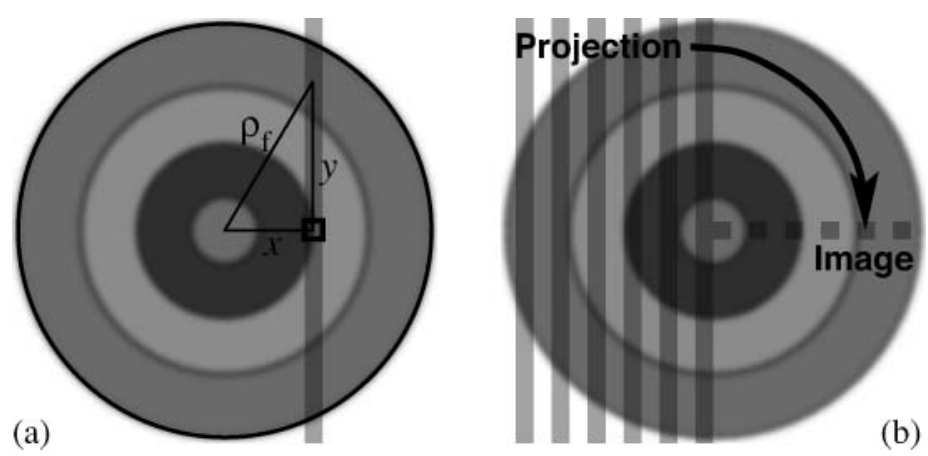

Figure 4. (a) Fiber cross-section irradiated by an X-ray beam at an offset $x$ from its center. The structure $\rho\left(\rho_{f}\right)$ shows fiber symmetry. From all structures along the beam path a superposition is probed. $y$ is the variable of the integration. (b) One-dimensional tomographic reconstruction turns the measured series of projected scattering patterns that carry the accumulated the structure information passed by the beam (vertical bars) into the image patterns from voxels (quadratic boxes) residing on the fiber radius.

either the spatial variation of the absorption in the plane, or of the scattering emanating from the resolved voxels in the plane. The smearing caused from projection is eliminated by application of the Fourier transform theory, and a clear image of the inner structure is obtained.

If the studied material shows cylindrical symmetry, the results of the measurement are no function of $\phi$ any more, and the complete image information is in a single microbeam scan. The fundamental geometry is sketched in Fig. 4a. The information in the measured signal $\{A\}(x)$ or $\{I\}(\mathbf{s}, x)$, respectively, does not represent the sought information $A(x)$ or $I(\mathbf{s}, x)$ originating from the small square (voxel) around the position $x$. Instead, to a first approximation it is represented by the projection integral

$$
\begin{aligned}
\{I\}(\mathbf{s}, x) & =2 \int_{x}^{\infty} I\left(\mathbf{s}, \sqrt{x^{2}+y^{2}}\right) d y \\
& =2 \int_{x}^{\infty} \frac{I\left(\mathbf{s}, \rho_{f}\right) \rho_{f} d \rho_{f}}{\sqrt{\rho_{f}^{2}-x^{2}}} .
\end{aligned}
$$

In the equation only the accumulated attenuation of the primary beam by X-ray absorption is not accounted for. This is no problem for very thin polymer parts with low absorption. For thicker samples the absorption correction from Section 2 is sufficient to account for it. The sought information in image space $\left(I\left(\rho_{f}\right)\right)$ along the radius $\rho_{f}$ of the fiber has to be reconstructed from the information in projection space $(\{I\}(x))$.

Equation (1) is the definition of the Abel transform. ${ }^{33}$ In X-ray scattering Eq. (1) is established textbook knowledge. ${ }^{4,34-39}$ There it describes the slit smearing. Even the inverse Abel transform

$$
\begin{aligned}
I(\mathbf{s}, x) & =-\frac{1}{\pi} \int_{0}^{\infty} \frac{d\{I\}\left(\mathbf{s}, \rho_{f}\right)}{d \rho_{f}} \frac{d y}{\rho_{f}} \\
& =-\frac{1}{\pi} \int_{x}^{\infty} \frac{d\{I\}\left(\mathbf{s}, \rho_{f}\right)}{d \rho_{f}} \frac{d \rho_{f}}{\sqrt{\rho_{f}^{2}-x^{2}}},
\end{aligned}
$$




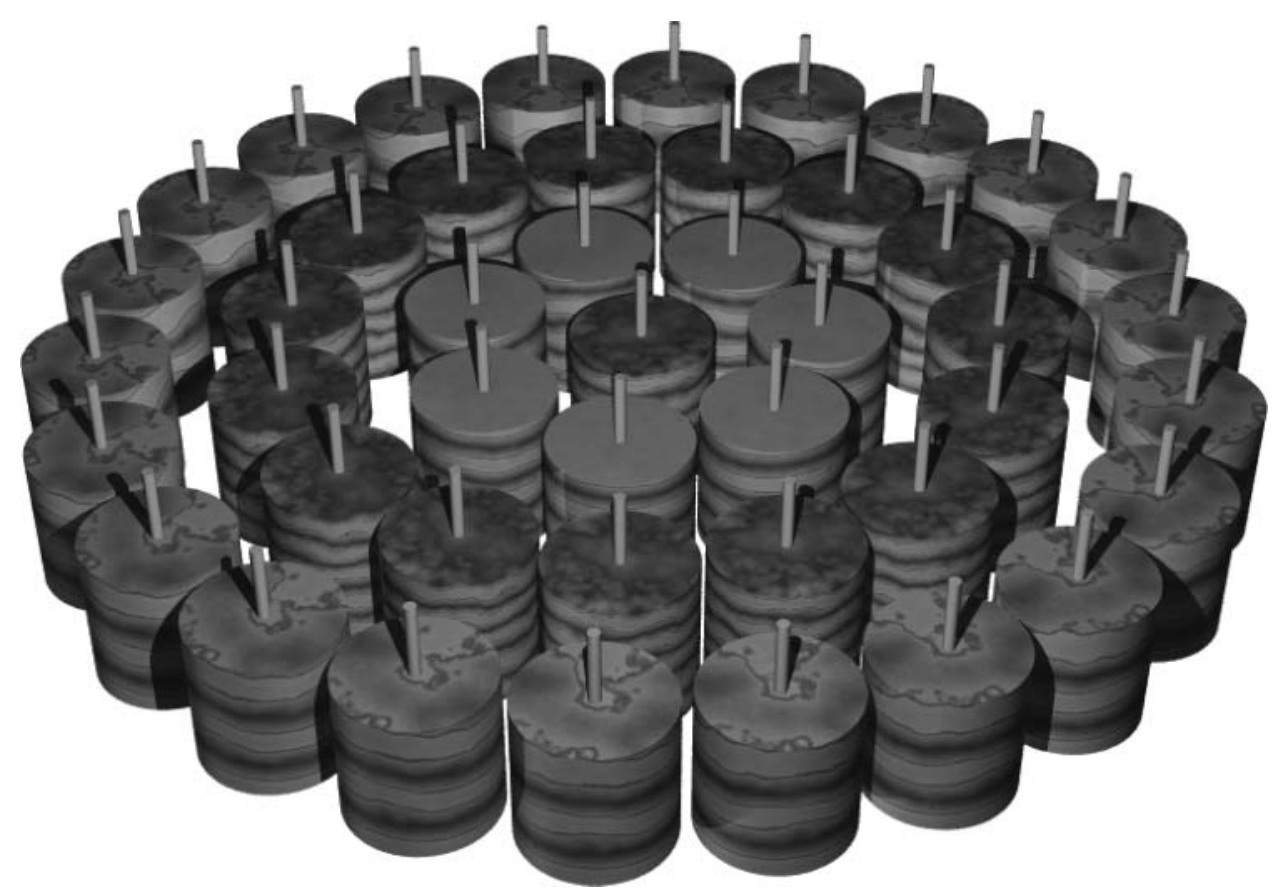

Figure 5. Sketch of local fiber symmetry inside the cross-section of the fiber. Each voxel has its own fiber axis. Inside the voxel there is only axial grain.

which first has been derived by Niels $\mathrm{Abel}^{40}$ is found in scattering textbooks since Guinier ${ }^{41}$ and DuMond. ${ }^{42}$ Similar to the filtered backprojection algorithm of the general tomography, low-noise reconstruction algorithms ${ }^{43-45}$ for the tomography of materials with cylindrical symmetry are readily available in the field of "one-dimensional tomography." The principle of one-dimensional tomographic reconstruction is sketched in Fig. 4b. It must be mentioned that here we implicitly assume that the scattering from every irradiated voxel in the fiber shows fiber symmetry itself (local fiber symmetry). Otherwise characteristic reconstruction aberrations are expected. ${ }^{28,46}$ The meaning of local fiber symmetry is sketched in Fig. 5. Deviations from local fiber symmetry (i.e. tangential or radial grain, resp. ${ }^{46}$ ) cause restricted or shifted visibility of scattering features along the fiber radius. These aberrations can be detected and result in additional information on the structure inside the fiber. ${ }^{46}$

\subsection{Application}

In an application-oriented feasibility study ${ }^{46}$ precursors of polymer microfibrillarreinforced composites (MFC) containing poly(ether)-block-amide (PEBA) and poly (ethylene terephthalate) (PET) with varying cold-draw ratio are studied. The studied strands are relatively thick, because presently the achievable "microbeam" at HASYLAB in Hamburg is relatively wide. The results from a direct analysis of the smeared measured patterns are compared to results obtained after tomographic reconstruction. Ideas for advanced practical applications of the XSF-CT method are discussed.

Data are presented from a cold-drawn (draw ratio $\lambda_{d} \approx 3$ ) co-extrudate of $70 \mathrm{wt} .-\%$ PEBA and 30 wt.- $\%$ PET (abbreviated: MFC73). In the scanning-microbeam experiment 

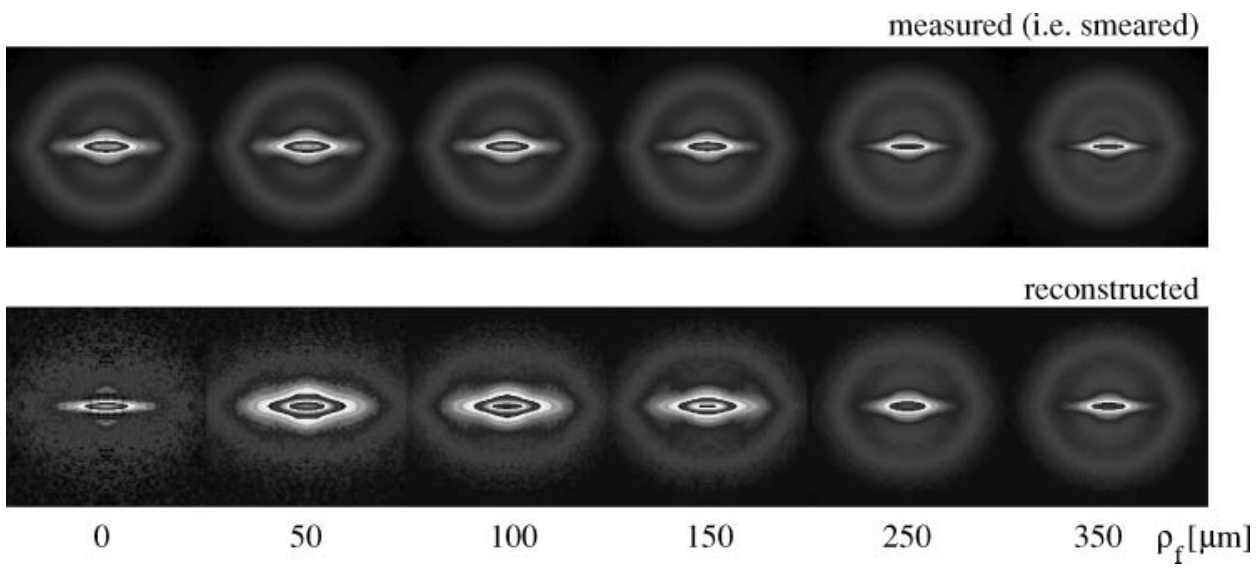

Figure 6. Cold-drawn $\left(\lambda_{d} \approx 3\right)$ MFC73 in a scanning microbeam experiment. Measured scattering intensity $\{I\}\left(s_{12}, s_{3}, \rho_{f}\right)$ (top row) and reconstructed scattering $I\left(s_{12}, s_{3}, \rho_{f}\right)$ (bottom row) for short distances $\rho_{f}$ from the fiber axis. The patterns display the range $-0.1 \mathrm{~nm}^{-1} \leq s_{12}, s_{3} \leq 0.1 \mathrm{~nm}^{-1}$ in uniform logarithmic scale $(s=(2 / \lambda) \sin \theta)$.

the strand shows an isotropic long period and an equatorial streak at almost every beam position (Fig. 6, top row). Only the tomographically reconstructed patterns (bottom row) exhibit that the long-period ring-reflection is not present in the core of the fiber. As the feature becomes visible, it first shows up at the equator. With increasing distance from the fiber axis, reflection arcs are growing towards the meridian. Above $\rho_{f}=300 \mu \mathrm{m}$ the $\operatorname{arcs}$ join into a closed circle. Because such behavior has been observed with neat PEBA as well, this orientation phenomenon is not indicating some interaction between the PEBA and the PET microfibrils. The reconstructed central voxel $\left(\rho_{f}=0\right)$ is dominated by one of the reconstruction aberration effects (from voxels with tangential grain). ${ }^{46}$ Examination of the equatorial streak exhibits only in the reconstruction that it grows broader towards the center of the fiber. The streak is allocated to needle-shaped domains, and is only observed with strands from co-extruded blends. If these needles are thin PET microfibrils, the tomography shows that in the center of the fiber these microfibrils are shorter than in an intermediate region. The structure gradient in the outer region of the strand is discussed in the original paper. $^{46}$

\section{SAXS Monitoring of Mechanical Tests}

\subsection{Motivation and Method Development}

Advanced polymer materials are urgently sought after e.g. in the automotive industry in order to accomplish the goals of climate protection by reduction of weight. Such newly engineered materials have to prove their serviceability in mechanical tests. Classical tensile tests are performed to determine the modulus and ultimate properties. Load cycling experiments are carried out to determine the fatigue behavior. In order to reveal the mechanisms of, e.g., failure or fatigue, it is desirable to monitor mechanical tests by SAXS. In this way the response of the nanostructure to mechanical load is revealed. Since about 2005 the advance of instrumentation at synchrotron beamlines facilitates considerable reduction of the exposure required for the recording of low-noise SAXS patterns. Such quality is 
required for a quantitative analysis by, e.g., the CDF method. ${ }^{47,48}$ Concerning the monitoring of tensile tests by SAXS this advance has meant that change-over from the stretch-hold technique ${ }^{49}$ to the more practical continuous straining has become possible, ${ }^{50}$ although the achievable strain rate is frequently still by a factor of 100 lower than that relevant in industry and service. With the advent of very high-brilliance synchrotron sources even this limitation is presently being abolished.

In order to gain full control on tensile and load-cycling tests a tensile tester has been built for operation at synchrotron beamlines. ${ }^{50}$ The control program written in LabVIEW ${ }^{\circledR}$ is continuously adapted to more complex experiments. Presently it can handle different kinds of continuous load-cycling programs that now are strictly synchronized with both the SAXS detector and a video grabber module (recording sequences of pictures of the sample with fiducial marks).

The latest methodical development ${ }^{51}$ is an automatic evaluation method of the video frames, by which the true macroscopic elongation of the sample can be determined with an accuracy of 3 decimals. Such high accuracy is required for thermoplastic materials that fail at low elongations (ca. 0.1). The method extracts the grating of the fiducial marks on the sample from the actual video image, computes the $1 \mathrm{D}$ correlation function, ${ }^{52,53}$ and evaluates its "long period."

\subsection{Results}

In a study ${ }^{50}$ of hard-elastic polypropylene (PP), scattering patterns recorded during continuous straining differ considerably from those recorded in the step-hold technique. Even though during exposure the elongation is no longer constant when applying the dynamic technique, the images collected in stretch-hold technique appear much more blurred (Fig. 7).

This result indicates relaxation of nanostructure while the extensometer stands still. Quantitative analysis shows that during relaxation the extension of crystalline lamellae

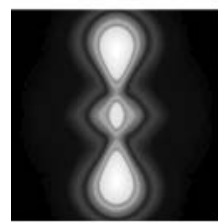

$\varepsilon=0$

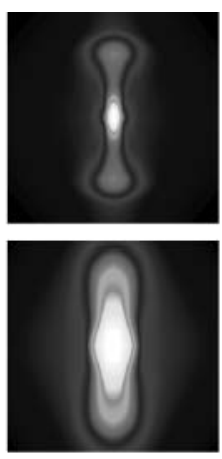

$\varepsilon=0.11$
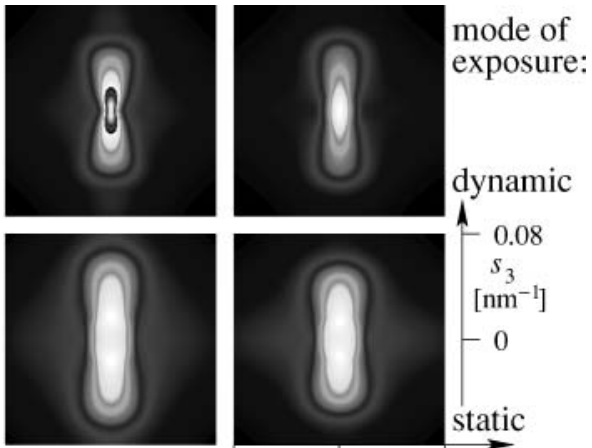

$-0.08$

$\varepsilon=0.23$
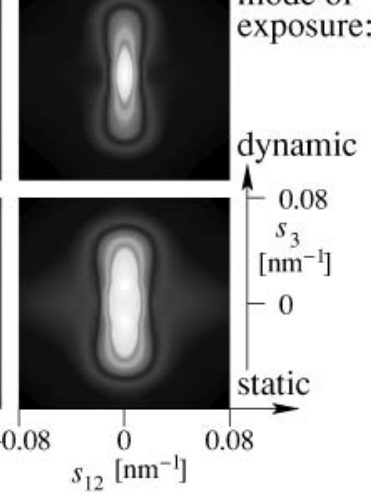

$\varepsilon=0.58$

Figure 7. Oriented PP films in tensile tests. Comparison between SAXS patterns $I\left(s_{12}, s_{3}\right)$ recorded during continuous straining (top row, $\dot{\varepsilon} \approx 10^{-3} \mathrm{~s}^{-1}$ ) and patterns from an experiment in stretch-hold technique (bottom row). Straining direction and meridian is vertical $\left(s_{3}\right)$. Equator $\left(s_{12}\right)$ is horizontal (C) Reproduced ${ }^{50}$ with permission of Wiley Interscience). 


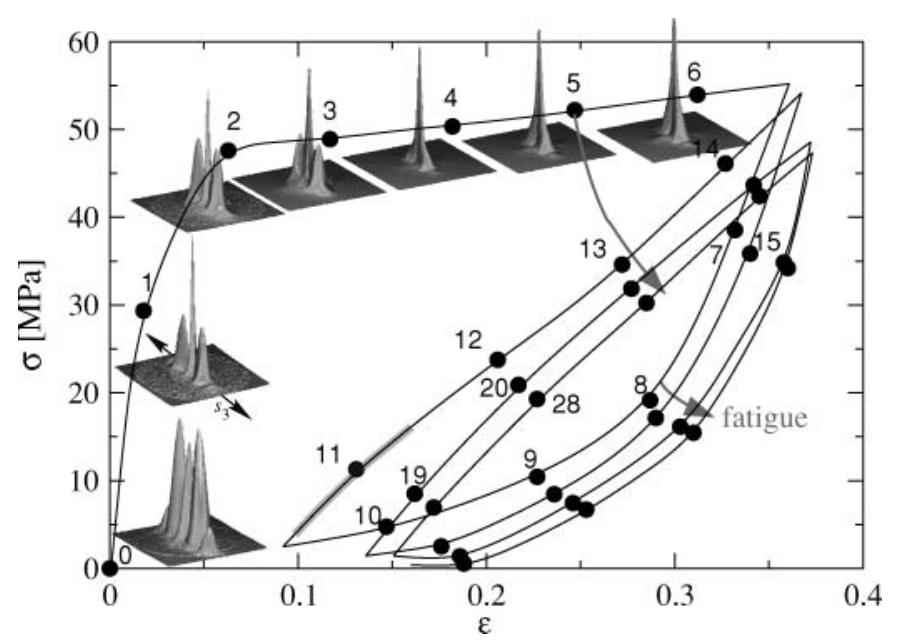

Figure 8. Oriented PP. Load cycling monitored by SAXS. Circular dots show where SAXS patterns have been recorded. Numerical labels indicate their sequence. The highlighted part of the curve near label 11 indicates the part traversed during the recording of pattern 11. The drawing direction with respect to the patterns, $s_{3}$, is indicated by a double-head arrow (C) Reproduced ${ }^{48}$ with permission of Wiley-VCH).

is increasing. Lamellae thicknesses are becoming non-uniform. The range of order is shortening. Cross-hatched lamellae are formed.

In another study ${ }^{48}$ slow continuous mechanical tests of oriented PP are monitored by SAXS and quantitatively evaluated by the CDF method. ${ }^{47}$ A continuous-strain test exhibits fracture and release of weak lamellae (2-10\% strain). Beyond that conversion of lamellae into needles is observed. As all layers are consumed, the material breaks. Fatigue is studied in a load-reversal experiment (between $10 \%$ and $35 \%$ strain, Fig. 8). In each cycle crystallization, layer break, and relaxation melting are observed. Figure 9 presents the result. The top chart shows the true elongation, $\varepsilon(t)$, imposed to the material and measured at the point of X-ray irradiation. The four load-reversal cycles are easily identified. The chart in the middle reports the extracted nanostructure parameters, $L(t)$, $e_{c a c}(t)$, and $S(t)$. The bottom diagram presents the macroscopic resistance, $\sigma(t)$, which the material is opposing to strain. The figure shows that crystallization, rupture of lamellae, and melting of fragments are continuously reshaping the domains of the PP material in the cycles of the fatigue test. The long-period cycle exhibits a phase shift with respect to the imposed strain cycling. Moreover, there is an indication of amplitude attenuation. Fatigue is demonstrated in the curve $\sigma(t)$ by decreasing stress peaks. An in-depth discussion of the zones indicated in Fig. 9 is in the original paper. ${ }^{48}$ In particular, the combination of SAXS and fatigue test shows the transition from stress-induced crystallization to crystallite rupture $\sigma(t) \approx 20 \mathrm{MPa}$.

In a recent study ${ }^{51}$ of microfibrillar reinforced blends based on polyethylene we have switched from hard-elastic materials to materials that fail at the low elongations typical for plastic polymers. Because of the much lower elongation at break $\left(\varepsilon_{b} \approx 0.1\right)$, now the macroscopic elongation $\varepsilon_{m}$ and nanoscopic elongation $\varepsilon_{n}$ must be determined with high precision. Respective methods are presented. The results show that the hardest materials exhibit a very inhomogeneous nanodomain structure. During straining their domains appear to be wedged together and inhibit transverse contraction on the nanometer scale. 


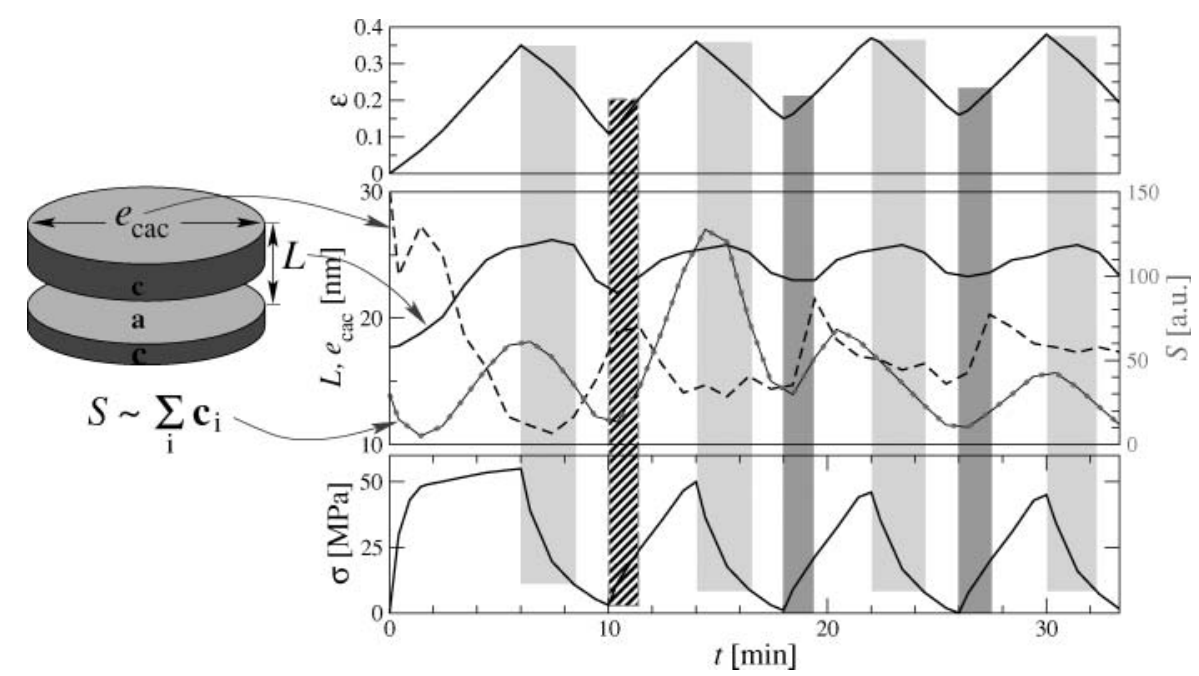

Figure 9. Dynamic load-reversal mechanical test of hard-elastic PP film. As a function of the elapsed time $t$ the macroscopic parameters elongation, $\varepsilon$ (top graph), and tensile stress, $\sigma$ (bottom graph) are displayed together with topological nanostructure parameters (middle). In the middle diagram the solid line shows the long period, $L$. The broken line displays the lateral extension, $e_{c a c}$, of a sandwich made from two crystalline lamellae. The line with circular dots exhibits the variation of the strength, $S$, of the CDF. Vertical bars indicate zones of strain-induced crystallization (dark bars) and relaxation-induced melting (light gray bars), respectively (C) Reproduced ${ }^{48}$ with permission of Wiley-VCH).

Further components are polyamides (PA6, PA12) (20-30\%) and as compatibilizer Yparex ${ }^{\circledR} 8102$ (YP) $(0-10 \%)$. Some HDPE/PA6 blends are additionally loaded with nanoclays (Nanomer ${ }^{\circledR}$ or Cloisite ${ }^{\circledR}$ ). Blending of HDPE with PA12 causes no synergistic effect. In the absence of nanoclay, PA6 and HDPE form a heterogeneous nanostructure with high Young's modulus. After addition of YP a more homogeneous scaffold structure is observed in which some of the PA6 microfibrils and HDPE crystallites appear to be rigidly connected, but the modulus has decreased. Both kinds of nanoclay induce a transition from a structure without transverse correlation among the microfibrils into a macrolattice with 3D correlations among HDPE domains from neighboring microfibrils. For extensions between $0.7 \%$ and $3.5 \%$ the scattering entities with $3 \mathrm{D}$ correlation exhibit transverse elongation instead of transverse contraction. The process is interpreted as overcoming a correlation barrier executed by the crystallites in an evasion-upon-approaching mechanism. During continued straining the 3D correlation is reduced or removed.

The true macroscopic elongation $\varepsilon_{m}$ is determined from video frames taken of the sample that carries fiducial marks (see Fig. 10a). Once for an experiment the user has to provide some input. It is based on the first image (Fig. 10a) of the series. The center of the $\mathrm{X}$-ray beam on the sample is marked by a cross in the image. Close to this center the user defines a rectangular region of interest (ROI), $\rho_{m}^{\prime}(x, y)$. In Fig. 10a this region is bordered by a dashed line. $x$ and $y$ are pixel coordinates in the direction of strain and perpendicular to it, respectively. The same ROI is applied to all video frames of the experiment. The ROI is structured by the fiducial marks running perpendicular to the straining direction. As is known from scattering theory, ${ }^{4}$ from such a structure function $\rho_{m}(x, y)$ the $2 \mathrm{D}$ correlation 


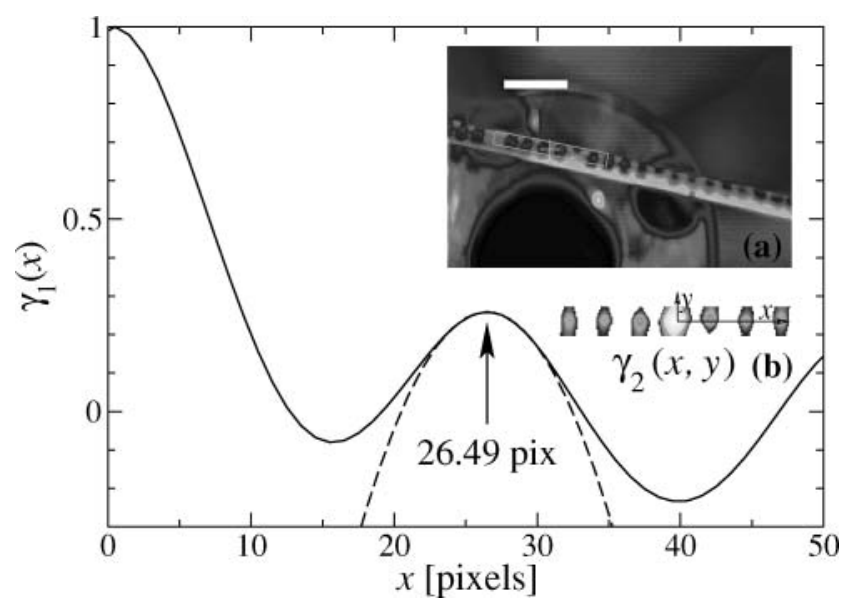

Figure 10. Elongation from recorded video frames. Inset a: In the first video frame a region of interest (ROI) with fiducial marks is defined. Inset $\mathbf{b}$ : From the ROI the $2 \mathrm{D}$ correlation function $\gamma_{2}(x, y)$ is computed. Main drawing: The center of the long-period peak in $\gamma_{1}(x)=\gamma_{2}(x, 0)$ is fitted by a parabola (dashed line) to compute the distance between the fiducial marks (C) Reproduced ${ }^{51}$ with permission of Wiley Interscience).

function

$$
\gamma_{2}(x, y)=\frac{\rho_{m}^{\star 2}(x, y)}{\rho_{m}^{\star 2}(0,0)}
$$

can be computed, with

$$
\rho_{m}(x, y)=\rho_{m}^{\prime}(x, y)-\bar{\rho}_{m}
$$

representing the fluctuation of $\rho_{m}^{\prime}(x, y)$ about its average $\bar{\rho}_{m}$, and the autocorrelation being defined by the integral

$$
f^{\star 2}(x, y)=\iint_{-\infty}^{\infty} f(u, v) f(u+x, v+y) d u d v .
$$

In Fig. $10 \mathrm{~b}$ the colored caps demonstrate, where $\gamma_{2}(x, y)$ is positive. The macroscopic elongation $\varepsilon_{m}$ in straining direction is the section $\gamma_{1}(x)=\left\lceil\gamma_{2}(x, y)\right\rceil_{1}(x)$ of $\gamma_{2}$ in straining direction. Figure 10 presents this curve and its analysis. Its first positive peak is the longperiod peak that is related to the actual average distance of the fiducial marks, $\ell$. A parabola (dashed line) is fitted to the long-period maximum, and the position of its vertex is determined (arrow). Thus, $\ell$ can be determined with an accuracy of 0.01 pixels. Let $\ell_{0}$ the initial distance between the marks, then the macroscopic elongation is $\varepsilon_{m}=\ell / \ell_{0}-1$.

Concerning the scattering patterns, similar analysis is possible. Nevertheless, the peaks observed in the scattering pattern or in the $\mathrm{CDFs}^{47}$ computed from the pattern are no longer one-dimensional but two-dimensional - positioned in the plane $\left(s_{12}, s_{3}\right)$ or $\left(r_{12}, r_{3}\right)$, respectively. Again, the user defines a ROI in which the analysis procedure searches for the peak maximum, extracts the peak cap, and fits now a bivariate ${ }^{54}$ polynomial of 2 nd order to it. From the coefficients of the polynomial several parameters are readily determined. Most important are the peak position and the widths of the peak in meridional and equatorial 

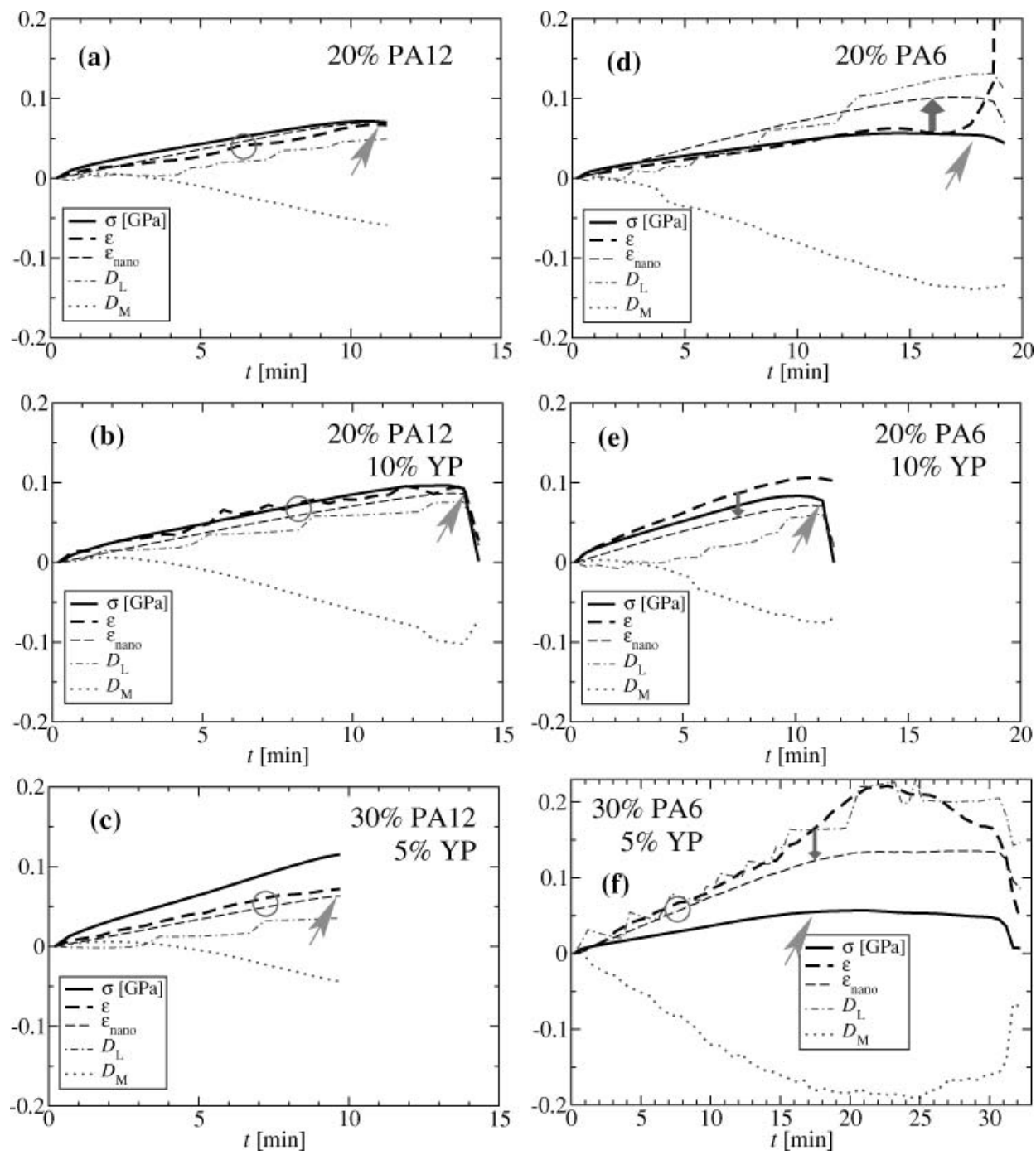

Figure 11. MFC precursor blends from HDPE, two different polyamides (PA12, PA6) and a compatibilizer (YP) in tensile tests. Evolution of macroscopic stress and strain $(\sigma, \varepsilon)$ as well as of nanostructure parameters. $\varepsilon_{\text {nano }}$ is the nanoscopic elongation computed from the HDPE long period. $D_{L}$ is the relative change of the width of the long period distribution. $D_{M}$ is the relative change of the extension of the microfibrils in transverse direction.

directions, respectively. Thus, the evolution of these parameters during the experiment can be tracked automatically.

Figure 11 presents the results of a quantitative nanostructure analysis for the blends which do not contain nanoclays. Elongations are illustrated by dashed lines. Bold lines show the macroscopic elongation, $\varepsilon$. Thin lines report the nanoscopic elongation $\varepsilon_{\text {nano }}$ of the HDPE matrix. Circular marks indicate regions in which $\varepsilon \approx \varepsilon_{\text {nano }}$. All materials reinforced by PA12 exhibit this similarity of macroscopic and nanoscopic deformation. Dashed-dotted lines show $D_{L}$, the relative variation of the breadth of the long-period 
distribution. The increase of all curves demonstrates increasing inhomogeneity of the long periods with increasing elongation. Dotted lines show $D_{M}$, the relative variation of the microfibrillar diameter during the straining process. In all materials the elongational deformation causes the microfibrils to thin. Variation of the material composition does not cause considerable changes. This is different with the samples reinforced by PA6 (Fig. 11d-f). Here an increase of the PA6 content to $30 \%$ causes strong thinning of the microfibrils. In the PA6 blends the strong transverse decrease is going along with only moderate nanoscopic elongation $\varepsilon_{\text {nano }}$ of the HDPE. An explanation for this finding could be microfibrillation by fracture of crystalline domains of the polyethylene. Moreover, the diagrams in Fig. 11d-f demonstrate a considerable difference (vertical arrows) between the two dashed curves. In Fig. 11e-f $\left(\varepsilon_{\text {nano }}<\varepsilon\right)$ the nanoscopic elongation of the HDPE phase is considerably lower than the macroscopic elongation. Similarity is only observed during the initial deformation in Fig. 11f (circular mark). In the 80/20 HDPE/PA6 blend (Fig. 11d) the nanoscopic elongation of the HDPE microfibrils is considerably longer than the macroscopic elongation $\left(\varepsilon_{\text {nano }}>\varepsilon\right)$. Although this finding appears to be unreasonable, an indication for a possible mechanism is in the strong increase of $D_{L}$ (Fig. 11d). This is discussed in the original paper. ${ }^{51}$

\section{Conclusions}

Considering the present instrumental development at synchrotron radiation facilities the development of advanced data evaluation methods appears to be both promising and necessary in order to master the future data flood. The three presented methods demonstrate the potential of such work. In order to discharge the user, a part of the data evaluation may be carried out at the synchrotron facility. Such added service would require not only considerable computing power, but also additional manpower. In addition to the beamline scientist an evaluation specialist would become necessary. It would be his job to detect if raw data must be smoothed. He would have to eliminate the machine background, would generate detector masks, would center and align each scattering pattern, and would fill blind areas from consideration of symmetry. As an added service, the community of the evaluation specialists could select some standard experiments for which complete user-friendly programming environments could be built. Nevertheless, for the predominant fraction of individually designed setups it will remain necessary for the polymer scientist himself to familiarize with adapted programming techniques. This will be of particular importance, if methods shall be developed that grow with the growth of instrumental capacity. Ultimately, it is expected that an increase of the quality of results returned from scattering experiments will be closely correlated to the manpower dedicated to the programming of data evaluation modules.

\section{Acknowledgment}

The author thanks the Hamburg Synchrotron Radiation Laboratory (HASYLAB) for beam time granted in the frame of project II-20080015. Development of the reported methods has been supported by the 7th framework program of the European Union (Project NANOTOUGH FP7-NMP-2007LARGE-2.1.1).

\section{References}

1. Broennimann, C.; Eikenberry, E. F.; Henrich, B.; Horrisberger, R.; Hülsen, G.; Pohl, E.; Schmitt, B.; Schulze-Briese, C.; Suzuki, M.; Tomizaki, T.; Toyokawa, A.; Wagner, A. "The PILATUS 1M detector," J. Synchrotron Rad., 2006, 13, 120-130. 
2. Hülsen-Bollier, G. The PILATUS 1 M Detector. A Novel Large Area Pixel Detector, Ph.D. thesis, Dept. Physics, University of Erlangen-Nürnberg, Germany 2005.

3. Stribeck, N. "Analysis of SAXS Fiber Patterns by Means of Projections," ACS Symp. Ser, 2000, $739,41-56$.

4. Stribeck, N. X-Ray Scattering of Soft Matter, Springer, Heidelberg, New York, 2007.

5. Stribeck, N. "Scattering of Soft Condensed Matter. From Fundaments to Application," In Applications of Synchrotron Light to Scattering and Diffraction in Materials and Life Sciences, Ezquerra, T. A.; Garciá Gutiérrez, M.; Nogales, A.; Gómez, M., eds., Springer, Berlin Heidelberg, 2009, volume 776 of Lect. Notes Phys. pp. 25-62.

6. Stribeck, N. "Deformation behavior of nanocomposites studied by X-ray scattering: Instrumentation and methodology," In Nano- and Micromechanics of Polymer Blends and Composites, Karger-Kocsis, J.; Fakirov, S., eds., Hanser Publisher, München, 2009, volume 1 pp. 269300.

7. VNI “PV-WAVE Manuals," V 7.5, Houston, TX, 2007.

8. Stribeck, N. "Downloads," http://www.chemie.uni-hamburg.de/tmc/stribeck/dl 2008.

9. Rajkumar, G.; AL-Khayat, H.; Eakins, F.; He, A.; Knupp, C.; Squire, J. "FibreFix-A new integrated CCP13 software package," Fibre Diffraction Rev., 2005, 13, 11-18.

10. Bian, W.; Wang, H.; McCullough, I.; Stubbs, G. "WCEN: a computer program for initial processing of fiber diffraction patterns," J. Appl. Cryst., 2006, 39, 752-756.

11. Stribeck, N. "On the determination of fiber tilt angles in fiber diffraction," Acta Cryst., 2009, A65, 46-47.

12. Franklin, R. E.; Gosling, R. G. "The structure of sodium thymonucleate fibres. II. Cylindrically symmmetrical patterson function," Acta Cryst., 1953, 6, 678-685.

13. Fraser, R. D.; Macrae, T. P.; Miller, A.; Rowlands, R. J. "Digital processing of fibre diffraction patterns," J. Appl. Cryst., 1976, 9, 81-94.

14. Stribeck, N.; Nöchel, U. "Direct mapping of fiber diffraction patterns into reciprocal space," J. Appl. Cryst., 2009, 42, 295-301.

15. Mencik, Z. "Crystal structure of isotactic polypropylene," J. Macromol. Sci. Phys., 1972, B6, 101-115.

16. Polanyi, M. "Das Röntgen-Faserdiagramm I. (The X-Ray Fiber-diagram I.)," Z. Phys., 1921, 7, 149-180.

17. Polanyi, M.; Weissenberg, K. "Das Röntgen-Faserdiagramm II. (The X-Ray fiber-diagram II.)," Z. Physik, 1923, 9, 123-130.

18. Stribeck, N.; Nöchel, U.; Funari, S. S. "Melting and crystallization of differently oriented sets of crystallites in hard-elastic polypropylene," Macromolecules, 2009, 42, 2093-2101.

19. Stribeck, N.; Nöchel, U.; Almendárez Camarillo, A.; Roth, S. V.; Dommach, M.; Bösecke, P. "SAXS study of oriented crystallization of polypropylene from a quiescent melt," Macromolecules, 2007, 40, 4535-4545.

20. Ruland, W. "X-ray determination of crystallinity and diffuse disorder scattering," Acta Cryst., 1961, 14, 1180-1185.

21. Dingenouts, N.; Bolze, J.; Potschke, D.; Ballauff, M. "Analysis of polymer latexes by small-angle X-ray scattering," Adv. Polym. Sci., 1999, 144, Epoxide Resins, Polyampholytes), 1-Epoxide Resins, Polyampholytes), 47.

22. Kieback, B.; Neubrand, A.; Riedel, H. "Processing techniques for functionally graded materials," Mater. Sci. Eng. A Struct. Mater. Prop. Microstruct. Proces., 2003, 362, 81-106.

23. Pompe, W.; Worch, H.; Epple, M.; Friess, W.; Gelinsky, M.; Greil, P.; Hempel, U.; Scharnweber, D.; Schulte, K. "Functionally graded materials for biomedical applications," Mater. Sci. Eng. A Struct. Mater. Prop. Microstruct. Proces., 2003, 362, 40-60.

24. Riekel, C.; Engström, P. "Diffraction and diffuse scattering from materials with microfocussed X-rays," Nuclear Instr. Meth. Phys. Res., 1995, B97, 224-230.

25. Waigh, T. A.; Donald, A. M.; Heidelbach, F.; Riekel, C.; Gidley, M. J. "Analysis of the native structure of starch granules with small angle x-ray microfocus scattering," Biopolymers, 1999, $49,91-105$. 
26. Kolb, R.; Wutz, C.; Stribeck, N.; V. Krosigk, G.; Riekel, C. "Investigation of secondary crystallization of polymers by means of microbeam X-ray scattering," Polymer, 2001, 42, 5257-5266.

27. Paris, O.; Li, C.; Siegel, S.; Weseloh, G.; Emmerling, F.; Riesemeier, H.; Erko, A.; Fratzl, P. "A new experimental station for simultaneous X-ray microbeam scanning for small- and wide-angle scattering and fluorescence at BESSY II," J. Appl. Cryst., 2007, 40, s466-s470.

28. Stribeck, N.; Almendarez Camarillo, A.; Nöchel, U.; Schroer, C.; Kuhlmann, M.; Roth, S. V.; Gehrke, R.; Bayer, R. K. "Volume-resolved nanostructure survey of a polymer part by means of SAXS microtomography," Macromol. Chem. Phys., 2006, 207, 1239-1249.

29. Bonse, U.; Busch, F. "X-ray computed microtomography $(\mu \mathrm{CT})$ using synchrotron radiation," Prog. Biophys. Molec. Biol., 1996, 65, 133-169.

30. Schroer, C. G.; Kuhlmann, M.; Roth, S. V.; Gehrke, R.; Stribeck, N.; Almendarez Camarillo, A.; Lengeler, B. "Mapping the local nanostructure inside a specimen by tomographic small-angle x-ray scattering," Appl. Phys. Lett., 2006, 88, 164102.

31. Schroer, C. G.; Kuhlmann, M.; Günzler, T. F.; Benner, B.; Kurapova, O.; Patommel, J.; Lengeler, B.; Roth, S. V.; Gehrke, R.; Snigirev, A.; Snigireva, I.; Stribeck, N.; Almendárez Camarillo, A.; Beckmann, F. "Full-field and scanning microtomography based on parabolic refractive x-ray lenses," Proc. SPIE, 2006, 6318, 6318H.

32. Kak, A. C.; Slaney, M. Principles of Computerized Tomographic Imaging; IEEE Press: New York, 1999.

33. Bracewell, R. The Fourier Transform and Its Applications; 3rd Ed. Mc Graw-Hill: New York, 1999.

34. Guinier, A.; Fournet, G. Small-Angle Scattering of X-Rays, Chapman and Hall: London, 1955.

35. Hosemann, R.; Bagchi, S. N. Direct Analysis of Diffraction by Matter; North-Holland: Amsterdam, 1962.

36. Alexander, L. E. X-Ray Diffraction Methods in Polymer Science; Wiley: New York, 1979.

37. Glatter, O.; Kratky, O., eds., Small Angle X-ray Scattering; Academic Press: London, 1982.

38. Feigin, L. A.; Svergun, D. I. Structure Analysis by Small-Angle X-Ray and Neutron Scattering; Plenum Press: New York, 1987.

39. Baltá Calleja, F. J.; Vonk, C. G. X-Ray Scattering of Synthetic Polymers; Elsevier: Amsterdam, 1989.

40. Abel, N. H. "Auflösung einer mechanischen Aufgabe," J. Reine Angew. Math., 1826, 1, 153-157.

41. Guinier, A.; Fournet, G. "Correction of measurments of low-angle X-ray scattering," Nature, 1947, 160, 501.

42. DuMond, J. W. M. "Method of correcting low angle X-ray diffraction curves for the study of small particle sizes," Phys. Rev., 1947, 72, 83-84.

43. Bitter, I.; Kaufman, A. E.; Sato, M. "Penalized-distance volumetric skeleton algorithm," IEEE Trans. Visualization and Computer Graphics, 2001, 7, 195-206.

44. Dasch, C. J. "One-dimensional tomography: a comparison of Abel, onion-peeling, and filtered backprojection methods," Applied Optics, 1992, 31, 1146-1153.

45. Dribinski, V.; Ossadtchi, A.; Mandelshtam, V. A.; Reisler, H. "Reconstruction of Abeltransformable images: The Gaussian basis-set expansion Abel transform method," Rev. Sci. Instr., 2002, 73, 2634-2642.

46. Stribeck, N.; Nöchel, U.; Fakirov, S.; Feldkamp, J.; Schroer, C.; Timmann, A.; Kuhlmann, M. "SAXS-fiber computer-tomography. Method enhancement and analysis of microfibrillarreinforced composite precursors from PEBAX and PET," Macromolecules, 2008, 41, 7637-7647.

47. Stribeck, N. "Extraction of domain structure information from small-angle X-ray patterns of bulk materials," J. Appl. Cryst., 2001, 34, 496-503.

48. Stribeck, N.; Nöchel, U.; Funari, S. S.; Schubert, T.; Timmann, A. "Nanostructure evolution in polypropylene during mechanical testing," Macromol. Chem. Phys., 2008, 209, 1992-2002.

49. Wu, J.; Schultz, J. M.; Yeh, F.; Hsiao, B. S.; Chu, B. "In-situ simultaneous synchrotron small- and wide-angle X-ray scattering measurement of poly(vinylidene fluoride) fibers under deformation," Macromolecules, 2000, 33, 1765-1777. 
50. Stribeck, N.; Nöchel, U.; Funari, S. S.; Schubert, T. "Tensile tests of polypropylene monitored by SAXS. Comparing the stretch-hold technique to the dynamic technique," J. Polym. Sci. Polym. Phys., 2008, 46, 721-726.

51. Denchev, Z.; Dencheva, N.; Funari, S. S.; Motovilin, M.; Schubert, T.; Stribeck, N. "Nanostructure and mechanical properties studied during dynamical straining of microfibrillar reinforced HDPE/PA blends," J. Polym. Sci. Part B: Polym. Phys., 2009, in print.

52. Vonk, C. G.; Kortleve, G. "X-ray small-angle scattering of bulk polyethylene," Colloid Polym. Sci., 1967, 220, 19-24.

53. Strobl, G. R.; Schneider, M. "Direct evaluation of the electron density correlation function of partially crystalline polymers," J. Polym. Sci., Part B: Polym. Phys., 1980, B18, 1343-1359.

54. Hall, E. L. Computer Image Processing and Recognition; Academic Press: London, 1980. 